\title{
Designing mHealth intervention for Women in Menopausal Period
}

\author{
Mirim Lee ${ }^{1}$, Bon-chang Koo ${ }^{1}$, Hee-seok Jeong ${ }^{1}$, \\ ${ }^{1}$ Human ICT convergence, H-lab \\ Sungkyunkwan University, \\ Suwon, Korea \\ Joongsing Park ${ }^{1}$, Juhee $\mathrm{Cho}^{2}$, Jundong Cho ${ }^{1}$ \\ ${ }^{2}$ Clinical Behabior \& Psycho-social Science \\ Sungkyunkwan University \\ Seoul, Korea \\ mirimrim@gmail.com, \{bubblek, jspaekkang, pjs6795, juheecho, jdcho\}@skku.edu
}

\begin{abstract}
Expected age of population is increasing as the quality of medical technology and services is improving. As a result, women nowadays are expected to spend $1 / 3$ of their lives after menopause. Precise knowledge of each of their menopausal stage and how to deal with corresponding symptoms would greatly increase the quality of their lives. There is a large amount of studies conducted on mHealth intervention, but the amount of study conducted for this particular target group is relatively small. In order to develop a mobile intervention for health management of menopausal women, this research set interviews with a focus group including middle aged women and stakeholders. As a result, we suggest a guideline for designing a health management system of menopausal women based on previous study and suggest a possible system that support women's wellness in menopausal period, with providing health information based on user's menopausal stage using mobile devices.
\end{abstract} factor

Keywords-menopaus; mHealth; intervention; women; human

\section{INTRODUCTION}

Expected age of people is increasing as the level of medical technology and healthcare service is improving. Currently, there are estimated 500 million people in the world that are over the age of 65[1]. Consequently, the social cost for agerelated healthcare service is increasing and the need for higher healthcare technology is imminent. There are many researches being conducted to enhance the quality of life and to save the medical cost for the elderly. Meanwhile, menopause is a physiological phase that all women experience at a certain age in their lifetime. Moreover, longer life expectancy has resulted in them experiencing a longer post-menopausal period-in average, women spend $1 / 3$ of their life in menopause [2]. Starting from menopausal transaction period, women face series of physical and mental challenges as estrogen level decreases[3.4]. The chances of cardiovascular disease, ostarthritis, and depression increase[5].However, although they experience such symptoms, they are not adequately examined because they lack the knowledge on the concept of and management for menopause $[6,7,10]$. It was found that the level of knowledge on menopause is positively correlated to the behavior to improve their health. Most maintained healthy lifestyle if they have adequate information [11]. Symptoms differ from each menopausal stage [8]. Therefore, woman in menopause needs to accurately understand her own menopausal stage and actively manage her health to efficiently minimize the corresponding symptoms. Also, It is widely believed that the people who is educated about the healthrelated condition will adhere to treatment plans and have improved health outcomes $[9,10]$.

PervasiveHealth community had been making efforts to improve the quality of life for various groups such as children, adolescents, elderlies and etc., but in truth researches conducted for the middle age women had been relatively inadequate. In this study, we present here the results of qualitative study consisting of interviews and focus group interviews with middle age woman and stakeholder. The aim of these investigation was to understand human factors of using mobile application to manage woman's health in menopause. We also aim to identify design consideration based on the user study and propose possible system design for mobile application for assisting woman in menopause.

\section{BACKGROUND WORK}

\section{A. Menopause and menopausal transaction period}

According to Stages of Reproductive Aging Workshop conducted by The American Society for Reproductive Medicine, menopausal transaction period starts around the age of 47 and lasts about 4 years. There are four stages of menopause; pre-menopausal period, early menopausal transaction period, late menopausal transaction period, and post-menopausal period [3]. Symptoms vary through women but most of them experience more than one of the following; hot depression, flushing, sweating, insomnia, and colpoxerosis[12]. Menopausal women experience slightly different symptoms depending on the stages they are in $[8,13]$.

\section{B. Recognition factors, actions, and attitude towards menopause}

Attitude affects the experience of menopause; positive attitude is followed by positive experience, and negative vice versa [14]. Further, severity of psychological and physical symptoms and knowledge level on menopause are shown to be affecting attitude. [11] Therefore, there should be a change of trend where their conditions are accurately measured and provided beforehand, so that they can positively react to the change and nurture the ability to control their behavior. [15] 


\section{C. mobile intervention for wellness of woman in menopause}

There already exists mobile applications for menopause management. This application lets the users to enter their menstrual dates and their symptoms such as hot flashes, night seats, loss of libido, and breast tenderness, and provides detailed information about the user's current health status. This application, though, merely shows statistically oriented selfreported information, and fails to inform the menopausal stage and provide interactive information for corresponding symptoms. Also, functions such as information exchange with peers who have same experience and health professionals must be added to support their mental wellness.

\section{STUDY DESIGN}

To research the experience of women in menopause, we recruited participants via a on-line menopause community. Participants were restricted to middle aged women aged between 45 and 60 who are experiencing menopause symptoms, or have experienced it within the last 5 years. 9 participants, of which 5 are employed and the other 4 are housewives, were recruited for the experiment. Additionally, we conducted interviews with 4 family members of both genders to understand their requests. We conducted semisturucted interview.

\section{A. Analysis Method}

We coded data from the interview and focus group interviews to look for functional requirements. The transcripts were coded using an iterative coding process. Potential codes created, around five core research themes: barriers, needs, expectation, information source, social support. Additionally, We asked questions for each of the functions, like as a calender, diary, alarm, etc., to check if they would be useful in there real-life experience.

We also included in the interview some issues regarding privacy.

\section{RESULTS}

Through the interview, the participants gave us clear descriptions on the menopause that they are experiencing.

\section{A. propensity to not record menstrual cycle.}

Most participants responded that they do not record their menstrual cycles on their calendars nor record dates anywhere else. They depended mostly on their memory to keep track of their menstrual cycles.

"My period was quite consistent at 25 days in between. And then it started skipping one month and the amount of bleeding started becoming irregular. ... ...when the period stops happening like that, I naturally sense that it might be the time for menopause."

The reason for their reluctance to record their period dates, they responded, was that unlike their past when they had to worry about pregnancy and contraception, they simply did not feel the necessity to do so.

"I am not at the age to concern about pregnancy so I did not really keep track of my cycles. When I was younger, ovulation day was important because of pregnancy and contraception, so I did check them, although not always. But I don't really need to now. I feel that I am going through menopause rather from the symptoms on my body than the irregular cycles."

\section{B. "Record your period day" Notification}

Some interviewees responded positively that notifications from personal smart mobile devices would be very useful in recording their period dates.

"I never recorded my cycles. But these days, I put all sorts of information on my cell phone anyway so I think it would be pretty easy to record my cycles on the phone."

"When you are in menopause, periods happen irregularly and if you are not reminded, you forget about it and lose track of them. I think notifications might be helpful."

\section{Lack of understanding on menopausal transition stage} Most of the participants had lack of understanding on menopausal transition stage. They were not aware that different symptoms appear in each stage. However, they responded that if the symptoms and management method are different for each stage, they would want to know all of them.

"I did not know such things existed. When symptoms appeared I just thought that was one of the things you go through when you are in menopause. It would be useful to have information like that. When symptoms are severe, they seem to be more important."

Some of them responded that, in their case, period stopped all of a sudden and never came back, that it would be hard to examine their menopausal transition stage.

"Others around me told me it happened slowly, but my periods happened every 30 days regularly and then it disappeared all of a sudden. I don't know if there really are such thing as transition stages."

\section{$F$. Exercise reminder and health management message}

Most participants answered that to manage the symptoms of menopause, they relied more on exercise and food intake change, rather than on medical treatments.

"I tried to start hiking more or walk a lot to make my feeling better"

For this reason, most were positive about exercise reminder.

"Once when symptoms were too severe, people suggested me to try hormonal therapy, but I wanted to try exercising because I didn't like the idea of chemical treatment. My husband took me out everyday for a walk and I think that helped a lot. Exercise reminder message on smartphones would be helpful as well, I think."

However, there were negative opinions regarding this function. "I don't know... for some people, it may be helpful, but I do not really pay much attention to cell phone notifications. Hmm... I think what you put on the message will be very important. People won't be encouraged by repetitive text notifications."

\section{G. Social Networking with others}


Majority of the participants answered that the most helpful thing in overcoming menopause was the conversation they had with their close friends.

"Whenever my symptoms occur, I talk to one of my friend who is 4 years older than me. She went through a menopause earlier than me so she gives me good advises. Also, I have seen her suffering severer symptoms in the past, so I feel better when I saw myself suffering similar but weaker symptoms. Having a conversation helped me a lot even when I was having a hard time emotionally."

\section{H.Family support}

Participants generally responded negatively to the idea of family support.

"You get easily annoyed when you are going through a menopause, and conflicts start happening. But I don't think having a direct conversation with them would really help the situation. They know I am in menopause, but I don't think there are much they can do to better the situation. I would prefer talking to my friend who has a similar experiences."

"I live with a husband and two sons. Would they ever able to understand something that would never happen to them? Also, wouldn't it seem over-reactive, since every woman experiences it? I feel more comfortable talking to my friend who share a similar experience."

On the other hands, Most family members want to provide help, but did not know how.

"I felt and knew that she was going through a tough time. When she gets annoyed at little things, I tried to ignore it as best as I can, but sometimes I got angry and back-fired at her. My friends and I had a conversation about their menopause, but we all did not really know what to do to help them."

Some answered that they tried to do something active with them.

"I think a regular exercise would be helpful for her mental health. So I took her out with me whenever I went out for an exercise. We talked a lot during walks and am trying to understand what she is going through"

\section{Privacy related}

Participants were not particularly worried about privacy issues when it comes to sharing their menopause information with their family members. They understood menopause as a stage that occurs naturally in every woman's life and did not feel much discomfort to talk about it. However, they would not bother talking about menopause to them because they do not think their family members are able to understand.

"I did not voluntarily come out with my menopause. It is not something to be ashamed of and they know it, too. But I just don't feel the need to talk to them anyway, also, because I really don't think telling them will make them understand. Do I need to?"

\section{J. Information source and its reliability}

Most women answered that they gain information from people they know. They also said they trust information coming from a closer friend more.

"I got to know many ways to manage my health while talking to my friends, from eating good food to exercising. When I was seriously considering a hormonal therapy, they told me it increases the risks of breast cancer so I turned to more natural ways to manage myself. They have experienced the same thing before and they are close to me so I trust them."

However, they only gained limited information from their peers and symptoms differ from person to person, and there were demands for professional and reliable source.

"I did not really enjoy talking about this subject. I was emotionally very weak. They would talk about their own stories and remedies but that did not help resolve my problems. I think a professional help from a psychologist would help a lot better, although I did not try that for myself."

\section{V.DESIGN CONSIDERSATION}

Based on the validation of our hypotheses of functions and requirements from interview and focus group interviews, we developed design consideration for a development of an effective mHealth for menopausal women' wellness. With this guideline in mind we suggest possible system architecture.

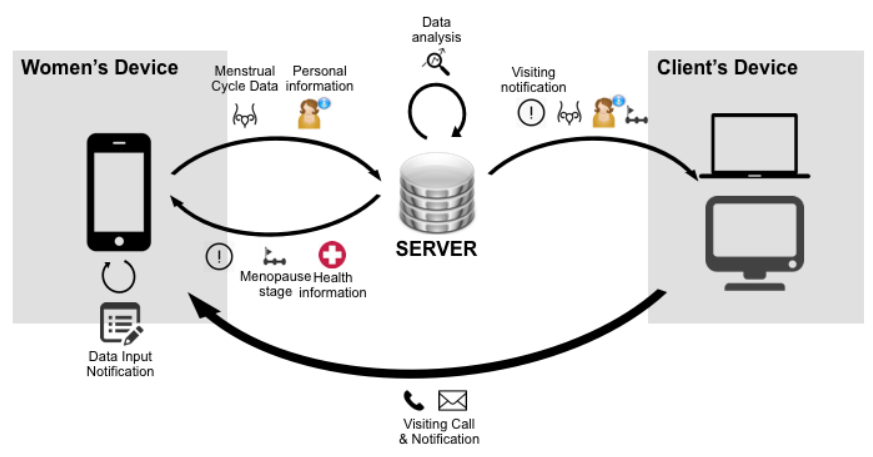

Fig 1. Potential System Architecture

- Build a solid UX mobile platform where users can easily record their menstrual cycle. The result shows that most women do not record their periods. However, Understanding where they are in a certain stage is important to manage their wellness. Therefore, It is important that they are encouraged to precisely record their period dates. Button at the first page that leads to recording menstruation date is a possible UI. Additionally, A pop-up message that asks according to their expectation period days(once a month), 'Did your period start today?' would appear.

- Let women with menopause in progress know what stage they are in. Current stage is assessed based on user's records. Majority of women were not aware of menopausal transition stages. It is essential that the application provide information based on a personalized data of their menstrual cycles. 
- Provide a personally tailored information that suits user's menopausal stage. Personalized health information for each stage is shown.

- Provide social support through SNS format. Most of the respondents answered that a conversation with their close friends were not only the most helpful in maintaining their mental health, it was also a biggest source of information. SNS function would be crucial in a developing a health management mobile application for the target group. A space where they can talk about their experiences should be prepared.

- Health, exercise recommendation by SMS messages. Since most participants wanted to self-manage this term other than receiving medical treatments, this application is required to send messages that would encourage exercising and better health management.

- Ensure the quality of information and service by cooperating with health professionals

- Don't have to involve the family members to manage their mental wellness. Support from family members were less important than we first speculated. Knowledge level of family members were helpful in health management but were not a significant source of emotional consolation. Also, family members should be educated on how to help their family member in menopause.

\section{CONCLUSION \& FUTURE WORK}

In this research, we attempted to understand what the barriers and needs are for menopausal women through related works and FGI. Based on this result, we came up with a possible prototype. This mobile-based health management system provides, first, SNS function that users can efficiently share their health information with others, second, message function that enables application to send text information on health management, third, reliable source from doctors, and fourth. The result of this study will be a guideline for the development of the actual application. However, this study has a few limits that are to be reissued in future works.

First, Most participants answered that they use smartphones. This is because smartphones are well distributed in South Korea. However, they did not seem particularly apt in maneuvering their smartphones. That is why additional studies on the user interface are required to have information ready with easier access. Second, Further study should be conducted to find what to include in those messages that would keep people interested and responsive. Third, Furthermore, prototype application from this research must develop based on the test with groups of women in menopause to evaluate its effectiveness. We will determine wheter this system is actually helpful in continuous health management by distributing the prototype application to the target group and executing longitudinal study. Finally, WHO recommends that mobile technology intervention designers for maternalchild health should be attuned to the socio-demographic, socio-cultural and socio-economic factors that influence female health behavior[12]. We should consider this issue for our future works.

\section{REFERENCES}

[1] Stanley Muravchic, Techology in an ageing society. Oxford Textbook of Anaesthesia for the Elderly Patient (2014), 26.

[2] Nursen Teoman, Ayse O. et al. The effect of exerciese on physical fitness and quality of life in postmenopausal women.The european menopuase jornal, Maturitas 47 (2004) 71-77

[3] Doran AT. Book extra: menopause and osteoporosis. People's Med Soc Newslett 1998;17(6):1-6

[4] Siegel HR. Primary prevention of postmenopausal osteoporosis. J Am Med Assoc 1998;280(21):1821-2.

[5] Vandenakker BC, Glass DD. Menopause and aging with disability. Phys Med Rehabil Clin North Am 2002;12(1):133-51.

[6] Polit,D.F.,\& Larocco, S.A.(1980). Woman's Knowledge about the menopause . Nursing Research , 29(1), 10-13.

[7] White, M.K., Martin, R.B., Yeaster, R.A ., Butcher, R.L.,\& Radin, E.L.(1984). The effects of exercise of on the bone of post - menopausal women . International Orthopeadics, 8, 209- 214.

[8] Soules MR, Sherman S, Parrott E. et al. Executive summary: stages of reproductive aging workshop (STRAW) Park City, Utah, July 2001. Menopause. 2001;8:402-7.

[9] Faustino R. et al.2004. An evaluation of the contents and quality of menopause information on the World Wide Web, Maturitas, pp. 276-82

[10] Fouzia R Memon, Leon Jonker, Roshan A Qazi, Knowledge, attitudes and perceptions towards menopause among highly educated Asian women in their midlife, Post Reprod Health December 2014 vol. 20 no. 4 138-142

[11] Mi-jeong Han, Ji-Hyun Lee, Factor Influencing Self-Identity and menopausal symptoms on Level of Depression in Middle Aged Women, Korean J Women Health Nurs Vol. 19, 275284,December, 2013mHealth: New horizons for health through mobile technologies. World Health Organization, Geneva, Switzerland, 2011.

[12] Groeneveld FPMJ, Bareman FP: Relationship between attitude towards menopause, well-being and medical attention among women aged 45-60 years, Matuiatas, 17, 1993, 77-88.

[13] Groeneveld FPMJ, Bareman FP: Relationship between attitude towards menopause, well-being and medical attention among women aged 45-60 years, Matuiatas, 17, 1993, 77-88.Hirsch, B., J. (1980) 'Natural Support Systems and Coping with Major Life Changes', American Journal of Community Psycholog, 8(2): 159-172.

[14] Bresnahan, M. J., \& Murray-Johnson, L. The Healing Web, Health Care for Women International 23(4): (2002) 398-407.

[15] Dennis R. Papini et al., Attitude Toward Menopause Among Married Middle-Aged Adults. Women\&Health. Web 21 Oct 2008 